\title{
Correction to: Numerical simulation and hydrodynamics analysis of a tethered underwater robot with control equipment
}

\author{
Shunyuan $\mathrm{Xu}^{1}{ }^{10} \cdot$ Jiaming $\mathrm{Wu}^{1} \cdot$ Haiyan $\mathrm{Lv}^{2} \cdot$ ChengHua $\mathrm{Ma}^{2} \cdot$ Xianyuan Yang ${ }^{1} \cdot$ Haotian Wang ${ }^{1} \cdot$ Yizhe Dou $^{1}$
}

Published online: 30 August 2021

(c) The Japan Society of Naval Architects and Ocean Engineers (JASNAOE) 2021

Correction to: Journal of Marine Science and Technology https://doi.org/10.1007/s00773-021-00839-5

The affiliations of third and fifth authors in the published paper are incorrect. The correct affiliation of third and fifth authors are available in this correction.

Publisher's Note Springer Nature remains neutral with regard to jurisdictional claims in published maps and institutional affiliations.

The original article can be found online at https://doi.org/10.1007/ s00773-021-00839-5.

Jiaming Wu

ctjmwu@ scut.edu.cn

Shunyuan Xu

517281577@qq.com

Haiyan Lv

lvhaiyan@ shunhaiship.com

ChengHua Ma

friedma@shunhaiship.com

Xianyuan Yang

yangxy_main@163.com

Haotian Wang

ouc1924@foxmail.com

Yizhe Dou

douyizhed1@163.com

1 Department of Naval Architecture and Ocean Engineering,

South China University of Technology, Guangdong province, Guangzhou 510640, China

2 Guangzhou Shunhai Shipyards Ltd., Guangdong province, Guangzhou 511440, China 